\title{
Riesgos Psicosociales en Conductores de Transporte de Carga y Pasajeros Urbanos e Interurbanos, y su Asociación con la Autopercepción de Salud y Siniestralidad Laboral
}

\section{PSYCHOSOCIAL RISKS IN FREIGHT AND PASSENGERS, URBAN AND INTER-CITY DRIVERS AND ITS ASSOCIATION WITH SELF-RATED HEALTH AND WORKPLACE ACCIDENTS}

\section{Helia Silva Bustos', Álvaro Lefio Celedón ${ }^{2}$, Nella Marchetti Pareto ${ }^{3}$, Philippe Benoit Marchetti ${ }^{4}$}

1. Médico cirujano. Especialista en Salud Pública. Magister en Salud Pública. Académica del Programa de Salud Ocupacional, Escuela de Salud Pública de la Universidad de Chile. Santiago, Chile.

2. Médico cirujano. Especialista en Salud Pública. Académico del Programa de Salud Ocupacional de la Escuela de Salud Pública de la Universidad de Chile. Santiago, Chile.

3. Ingeniero Químico. Jefe de Programa de Salud Ocupacional de la Escuela de Salud Pública de la Universidad de Chile.

4. Ingeniero Civil Industrial. Consultor Independiente en Gestión de Personas y Desarrollo Organizacional.

\section{RESUMEN}

La exposición a factores de riesgo psicosociales presentes en el lugar de trabajo constituye un tema prioritario para la salud ocupacional. El objetivo del estudio es estimar la asociación entre factores de riesgo psicosocial presentes en el trabajo, y autopercepción de salud y siniestralidad laboral, en conductores de vehículos de transporte de carga y pasajeros, urbanos e interurbanos, de empresas afiliadas a la Asociación Chilena de Seguridad.

Se aplicaron 4 cuestionarios a una muestra de 356 conductores de 4 empresas de transporte, en que $100 \%$ fue hombre con una edad promedio de 45,4 \pm 9,7 años; el 70,8\% transporta pasajeros, con jornadas de 11,4 horas/día. Todas las dimensiones de ISTAS-21 resultaron con promedios inferiores a la referencia salvo "trabajo activo y desarrollo de habilidades". El 93,8\% se reportó como "sin alteraciones de salud mental”. Se demostraron asociaciones estadísticamente significativas entre dimensiones del ISTAS 21 con, GHQ-12 y participación en accidentes de tránsito (sólo compensaciones), aun después de ajustar por variables de confusión.

Esto permitió el diseño de intervenciones, consensuadas con las empresas, a fin de controlar/eliminar estas exposiciones para mejorar la salud y calidad de vida de los trabajadores y reducir las enfermedades profesionales y accidentes del trabajo relacionados.

(Silva H, Lefio A, Marchetti N, Benoit P, 2014. Riesgos Psicosociales en Conductores de Transporte de Carga y Pasajeros Urbanos e Interurbanos, y su Asociación con la Autopercepción de Salud y Siniestralidad Laboral. Cienc Trab. May-Ago; 16 [50]: 67-74).

Palabras Clave: RIESGO LABORAL, ESTRÉS PSICOSOCIAL LABORAL, FACTORES PSICOSOCIALES.

\section{ABSTRACT}

Exposure to psychosocial risk factors present in the workplace is a priority topic for occupational health. The aim of the study is to estimate the association between psychosocial risk factors present at work, and self-rated health and occupational accidents in drivers of passenger and freight transport, urban and intercity, of companies affiliated with the Chilean Safety Association.

Four questionnaires were applied to a sample of 356 drivers from 4 Transport companies, in which $100 \%$ were male with a average age of $45,4 \pm 9,7$ years; $70,8 \%$ carry passengers, with working days of 11,4 hours / day. All dimensions of ISTAS-21 resulted with lower averages to the reference except for "active job and skill development." 93,8\% was reported as "no mental health disorders". Statistically significant associations between dimensions of ISTAS 21, GHQ-12 and involvement in traffic accidents (just compensation), even after adjusting for confounding variables were demonstrated.

This allowed the design of interventions, agreed with the companies, in order to control/eliminate these exposures to improve the health and quality of life of workers and reduce occupational diseases and work-related accidents.

Key words: OCCUPATIONAL HAZARD, OCCUPATIONAL PSYCHOSOCIAL STRESS, PSYCHOSOCIAL FACTORS.
Correspondencia / Correspondence:

Helia Silva Bustos

Escuela de Salud Pública, Facultad de Medicina, Universidad de Chile Independencia 939, Santiago, Chile

Tel.: (56-2) 978 6146 / 9782978

e-mail: helia_s@yahoo.com

Recibido: 28 de Junio 2014 / Aceptado: 10 de Julio 2014

\section{INTRODUCCION}

Un desafío actual y global en el campo de la salud ocupacional corresponde a la identificación, cuantificación, evaluación y posterior intervención respecto de la exposición laboral a factores de riesgo psicosociales presentes en el lugar de trabajo, entendidos actualmente como "conjunto de condiciones relacionadas con la organización y contenido de las tareas, con los procedimientos y métodos de trabajo, asi como con las relaciones entre los trabajadores y con sus superiores, cuya exposición prolongada en el 
tiempo aumenta la posibilidad de experimentar tensión psíquica la que, una vez acumulada residualmente, será un precursor de los trastornos o problemas de salud y afectará la productividad. Por tanto están estrechamente ligados al ausentismo, motivación y desempeño". ${ }^{1}$

Entre las principales características de los factores de riesgo psicosociales (FRPS) se encuentran ${ }^{1}$ : a) se extienden en el espacio y en el tiempo; b) exhiben dificultad en su objetivación; c) afectan a otros riesgos presentes en el trabajo; d) tienen escasa cobertura legal; e) están moderados por otros factores; $y, f$ ) presentan dificultad en su intervención.

Son agentes con alta probabilidad de afectar negativamente a la salud y el bienestar del trabajador ${ }^{2}$ actuando como desencadenantes de estrés, aunque sus efectos puedan ser expresados de diferente modo acorde a la vulnerabilidad del sujeto expuesto. ${ }^{3}$ El estrés laboral se define como "un patrón de reacciones emocionales, cognitivas, fisiológicas y de comportamiento a ciertos aspectos adversos o nocivos del contenido del trabajo, organización del trabajo y el medio ambiente de trabajo. Es un estado que se caracteriza por altos niveles de excitación y de respuesta y la frecuente sensación de no poder afrontarlos". 4

Producto de la facilidad de exposición en entornos laborales y sus consecuencias sobre la salud de los trabajadores es que estos factores se han estudiado desde fines de 1970, momento en que se asociaron a la generación de estrés en el trabajador. En este sentido, destaca el Modelo de Karasek ("alta demanda-bajo control") ${ }^{5,6}$ caracterizado por dar cuenta de una alta demanda psicológica y baja posibilidad de decisión, siendo tanto la demanda como el control parte de las condiciones laborales. Este modelo cuenta con abundante apoyo empírico al demostrar su asociación con enfermedad cardiovascular ${ }^{7}$, patologías músculo-esqueléticas ${ }^{8}$, y psiquiátricas, ${ }^{9,10}$ trastornos depresivos, suicidios y conductas parasuicidas, junto a trastornos de estrés posttraumático asociados a violencia, acoso laboral y sexual.

Los modelos de "apoyo social en el trabajo" de J. Johnson, y el de "desbalance esfuerzo-recompensa" de Siegrist también han demostrado los efectos cardiovasculares secundarios a esta exposición. ${ }^{11}$

$\mathrm{Si}$ bien este agente de riesgo laboral específico afecta transversalmente a los trabajadores de todos los sectores económicos, el rubro transporte es de especial importancia, puesto que se han reportado altos índices de estrés en los conductores, quienes tienen la responsabilidad de movilizar personas o cargas durante jornadas laborales que se caracterizan por tener turnos rotativos e incluir la noche.

Los resultados de la Encuesta de Población Activa de la Unión Europea del 2007 muestra que los trabajadores que ven afectada su salud mental corresponden al 30\% de quienes laboran en los sectores de "transporte, almacenamiento y comunicaciones, intermediación financiera, administración pública y defensa”, mismos rubros en que la prevalencia de estrés, depresión o ansiedad fue más alta. ${ }^{12}$

La Agencia Europea para la Salud y Seguridad en el Trabajo al año $2011^{13}$ constata que los conductores del transporte terrestre son los más afectados por estrés laboral al compararlos con otras actividades económicas (33,3\% versus 23,7\%).

Los transportistas perciben que su trabajo requiere un alto nivel de concentración y que no tienen control sobre las actividades realizadas. ${ }^{14}$ Los altos índices de estrés laboral en operadores del transporte urbano se han asociado a tensión, fatiga y carga mental ${ }^{15}$, los cuales han sido demostrados incluso a través de respuestas neuroendocrinas ${ }^{16}$ con elevados niveles de cortisol $^{17}$, y se han relacionado a la ocurrencia de accidentes menores ${ }^{18}$. Se ha reportado que padecen de Burnout severo en un 3,6\% y en nivel medio en un 30,1\%. ${ }^{19}$
El estrés autorreportado en transportistas también se ha asociado a un mayor nivel de consumo de alcohol ${ }^{20}$ y a ausentismo laboral ${ }^{21,22}$, a una mayor ocurrencia de accidentes laborales y enfermedades profesionales $^{23}$, y a elevados índices de violencia que han sido determinados sobre sus jornadas laborales (hasta cuatro veces más que otros rubros). ${ }^{24}$ De hecho, Obelenis reporta que el 46\% de los trabajadores del rubro de transporte de Lituania fuman y que los bebedores de alcohol alcanzan el 83,8\%. Se demostró una asociación estadisticamente significativa entre alteraciones del sueño, cefalea y mal humor con el estrés experimentado en el trabajo $(\mathrm{p}=0,002) \mathrm{y}$ que una jornada de trabajo más de 8 horas estaba estrechamente relacionada con el mal estado de salud $(p<0,05)$ y estrés en el trabajo ( $\mathrm{p}<0,001)$.

Bongers et al. ${ }^{25}$ demostraron que: presión del tiempo, trabajo monótono, alta carga de trabajo, bajo control de empleo y falta de apoyo social por parte de los compañeros se correlacionan positivamente con trastornos músculo-esqueléticos.

En Chile, La Primera Encuesta Nacional de Empleo, Trabajo, Salud y Calidad de Vida de los Trabajadores y Trabajadoras en Chile (ENETS 2009-2010) ${ }^{26}$ da cuenta, entre sus principales resultados que los hombres presentan más riesgo de accidentarse en el trabajo, y éstos son de mayor gravedad; y que los trabajadores informales y con inestabilidad laboral refieren comparativamente peor situación de salud respecto al año anterior que los otros grupos.

La investigación de Pérez-Franco señala que el trabajo en general se caracteriza por ser de alta tensión y con un desbalance del esfuerzorecompensa. Tras analizar los datos de la ENETS 2009-2010 y modelar las dimensiones de riesgo psicosociales según el Cuestionario SUSESO ISTAS-21, concluye que el rubro transporte se posicionó en el $9^{\circ}$ lugar con una media de prevalencia de exposición de 32,7 puntos, sin diferencia significativa según sexo.

Olivares ${ }^{28}$ demostró una prevalencia de Burnout en conductores del transporte público (Transantiago) de 23,2\% y de Burnout clínico de un 6,3\%. Asimismo, determinó un coeficiente de correlación de Pearson de 0,351 entre la Carga Mental y Burnout, considerando a la carga mental como un buen predictor de este sindrome (relación positiva, significativa y moderada).

Respecto de la fatiga laboral y su asociación con la ocurrencia de accidentes laborales en conductores del rubro de transporte, Neisa ${ }^{29}$ determina en su estudio que el 60\% de los conductores de carga pesada que sufrió accidentes laborales tenía "fatiga". Kaur ${ }^{30}$ relaciona la fatiga laboral con la carga laboral debido a: a) el número excesivo de horas de trabajo, b) el número inadecuado de horas de sueño, c) la conducción nocturna, $y$ d) los horarios irregulares de trabajodescanso.

Frente a este escenario, este estudio estima la asociación entre factores de riesgo psicosociales presentes en el trabajo, y autopercepción de salud y siniestralidad laboral, en conductores de vehículos de transporte de carga y pasajeros, urbanos e interurbanos, de empresas afiliadas a la Asociación Chilena de Seguridad.

\section{MATERIAL Y MÉTODOS}

\section{Corresponde a un diseño transversal analítico}

La población correspondió a conductores de transporte de carga y pasajeros, mayores de 18 años, de ambos sexos, que se desempeñan en zonas urbanas e interurbanas, con más de seis meses trabajando en la empresa y que estas últimas se encontraran afiliadas a la Asociación Chilena de Seguridad (ACHS) como 
Organismo Administrador de la Ley 19.664, durante los años 2012 y 2013.

Para el reclutamiento de las posibles empresas participantes, se contó con la base de datos de la ACHS al año 2012, la cual se filtró por zona y por rubro “transporte”. De las 40 empresas contactadas sólo tres accedieron participar, las otras o no aceptaron o no informaron su decisión en un plazo amplio determinado. Una última empresa se contactó a fin de ser incluida en el estudio, totalizando así 4 empresas participantes.

Mediante muestreo aleatorio simple, utilizando criterios estadísticos para una proporción en población finita y asumiendo un error del 5\%, se obtuvo un tamaño muestral de 252. Dado que la última empresa incorporada contaba con más de 1.000 trabajadores, al calcular un muestreo representativo por faena se alcanzó la cifra total de 356 participantes, con una tasa de respuesta del 100\%.

La aplicación de los cuestionarios fue de carácter mixto: colectiva, realizada en grupos pequeños y, también individual.

A los trabajadores presentes los días de visita acordados por cronograma junto a la empresa, que aceptaron participar y que consintieron por escrito, se les solicitó completar los cuestionarios anónimos mediante autorreporte.

Los cuestionarios aplicados, en un tiempo que fluctuó entre los 30 y 45 minutos, fueron:

- Cuestionario Sociodemográfico

- Cuestionario ISTAS-21 (Versión Media) ${ }^{31}$ : Consta de 85 preguntas breves con 5 opciones de respuesta, con puntuación de 0 a 4 . Las variables dan cuenta de 21 sub-dimensiones agrupadas en 5 dimensiones. Se estandarizaron las 21 escalas y se establecieron para cada una tres puntos de corte ("más favorable para la salud" o verde, "intermedio" o amarillo, y "más desfavorable para la salud" o rojo) a partir de la distribución en terciles de las puntuaciones.

- Cuestionario de Salud General (GHQ-12): desarrollado por Goldberg $^{32}$ y validado en nuestro país ${ }^{33,34,35}$, cuyo objetivo es detectar morbilidad psicológica y posibles casos de trastornos psiquiátricos, como screening y no como diagnóstico.

- Cuestionario de Siniestralidad laboral de tránsito: diseñado para capturar datos referentes a datos referentes al número de accidentes de tránsito en los cuales ha participado directa o indirectamente siendo conductor de esta empresa, cuántos de ellos han sido graves y cuántos fatales.

\section{Plan de análisis de los datos}

Los datos recopilados fueron ingresados a una máscara de EpiData (versión 3.1). El análisis exploratorio permitió conocer a priori la información (errores, outliers, missing).

En cuanto a las exposiciones a factores de riesgo psicosociales presentes en el lugar de trabajo se determinaron las prevalencias de las 21 sub-dimensiones. Posteriormente, se estandarizaron los puntajes de las escalas crudas de estas sub-dimensiones para que todas oscilaran entre 0 y 100, valores que finalmente fueron agregados en 5 dimensiones; esto quiere decir que el valor obtenido mientras sea más cercano a 100, la prevalencia de exposición es más alta. En base a esto se construyeron los terciles.

Para evaluar la asociación entre la presencia de factores de riesgo psicosocial presentes en el trabajo $\mathrm{y}$, su percepción de salud $\mathrm{y}$ siniestralidad, se utilizó estadística paramétrica y no paramétrica, según la distribución de las variables, además de modelos multivariados lineales y logísticos. Los análisis de asociación, utilizando
Tabla 1.

Características de los trabajadores del estudio.

\begin{tabular}{|c|c|c|c|c|c|}
\hline \multirow[b]{3}{*}{ Empleados (n) } & \multicolumn{4}{|c|}{ Empresa } & \multirow{2}{*}{ Total } \\
\hline & 1 & 2 & 3 & 4 & \\
\hline & 78 & 26 & 84 & 168 & 356 \\
\hline \multicolumn{6}{|c|}{ Caracteristicas Sociodemográficas } \\
\hline Edad (años) & $41,9 \pm 10,0$ & $44,1 \pm 9,5$ & $45,5 \pm 11,0$ & $47,2 \pm 8,4$ & $45,4 \pm 9,7$ \\
\hline \multicolumn{6}{|c|}{ Último nivel educacional aprobado $(\%)$} \\
\hline Ed. Básica & 16,6 & 15,4 & 1,2 & 7,7 & 8.7 \\
\hline Ed. Media & 64,1 & 69,2 & 66,7 & 76,2 & 70,8 \\
\hline Ed. Técnica & 16,7 & 7,7 & 30,9 & 15,5 & 18,8 \\
\hline Ed. Universitaria & 2,6 & 7,7 & 1,2 & 0,6 & 1,7 \\
\hline \multicolumn{6}{|l|}{ Estado Civil (\%) } \\
\hline Soltero & 7,7 & 7,7 & 19,0 & 5,9 & 9,5 \\
\hline Casado & 57,7 & 57,7 & 52,4 & 63,1 & 59,0 \\
\hline Separado de hecho & 7,7 & 11,5 & 9,5 & 6,6 & 7,9 \\
\hline Conviviente & 26,9 & 23,1 & 16,7 & 19,6 & 20,8 \\
\hline Viudo & 0 & 0 & 2,4 & 1,2 & 1,1 \\
\hline Divorciado & 0 & 0 & 0 & 3,6 & 1,7 \\
\hline \multicolumn{6}{|c|}{ Caracterización Ocupacional General } \\
\hline \multicolumn{6}{|c|}{ Tipo de Transporte (\%) } \\
\hline De Pasajeros & 0 & 0 & 100 & 100 & 70,8 \\
\hline De Carga & 100 & 100 & 0 & 0 & 29,2 \\
\hline \multicolumn{6}{|l|}{ Zona de trabajo $(\%)$} \\
\hline Radio urbano & 29,5 & 69,2 & 8,3 & 7,7 & 17,1 \\
\hline Interurbano & 37,2 & 11,5 & 8,3 & 22,6 & 21,6 \\
\hline Rural & 2,6 & 15,4 & 0 & 2,4 & 2,8 \\
\hline Difícil acceso & 3,8 & 0 & 2,4 & 47,0 & 23,6 \\
\hline Mixto & 26,9 & 3,9 & 81,0 & 20,2 & 34,8 \\
\hline \multicolumn{6}{|c|}{ Trabajo se corresponde con salario (\%) } \\
\hline Si & 42,3 & 34,6 & 15,5 & 44,9 & 36,6 \\
\hline \multicolumn{6}{|l|}{ No, trabajo es } \\
\hline \multicolumn{6}{|l|}{ No, trabajo es } \\
\hline menos que salario & 2,6 & 0 & 3,5 & 0,6 & 1,7 \\
\hline No lo sé & 9,0 & 11,5 & 2,4 & 1,8 & 4,2 \\
\hline \multicolumn{6}{|c|}{ Antelación de comunicación para cambio de horario (\%) } \\
\hline No cambian horario & 56,4 & 76,9 & 20,2 & 37,9 & 40,7 \\
\hline Comunican con & & & & & \\
\hline varios dias & 7,7 & 3,9 & 27,4 & 19,9 & 17,8 \\
\hline Comunican con & & & & & \\
\hline algunos dias & 3,8 & 7,7 & 11,9 & 5,4 & 6,8 \\
\hline Comunican el día antes & 16,7 & 0 & 11,9 & 22,9 & 17,2 \\
\hline Comunican el mismo dia & ía 15,4 & 11,5 & 28,6 & 13,9 & 17,5 \\
\hline \multicolumn{6}{|l|}{ Horario de trabajo (\%) } \\
\hline Mañana y tarde & 42, & 38,53 & 8,3 & 49,4 & 37,4 \\
\hline Mañana & 2,6 & 0 & 0 & 1,2 & 1,1 \\
\hline Tarde & 0 & 0 & 1,2 & 0 & 0,3 \\
\hline Rotativo sin noche & 5,1 & 0 & 0 & 9,5 & 5,6 \\
\hline Rotativo con noche & 0 & 0 & 70,3 & 20,8 & 26,4 \\
\hline Irregular & 46,2 & 19,2 & 13,1 & 9,6 & 19,1 \\
\hline Otro & 3,8 & 42,3 & 7,1 & 9,5 & 10,1 \\
\hline \multicolumn{6}{|l|}{ Horas trabajadas } \\
\hline al dia (horas) & $11,6 \pm 1,9$ & $11,2 \pm 2,8$ & $12,4 \pm 3,4$ & $10,7 \pm 4,6$ & $11,4 \pm 3,8$ \\
\hline \multicolumn{6}{|l|}{ Horas trabajadas a la } \\
\hline semana (horas) & $67,4 \pm 12$ & $65,5 \pm 14,8$ & $54,4 \pm 10,1$ & $41,4 \pm 30$ & $51,9 \pm 24,8$ \\
\hline \multicolumn{6}{|c|}{ Años de Conducción $(\mathrm{Me}(R I C))^{*}$} \\
\hline En la vida en total & $15(8-22)$ & $13,5(9-23)$ & $20(12-30)$ & $22(18-27,5)$ & 5) $20(12-26)$ \\
\hline En la empresa & $4(2-8)$ & $5,5(5-6)$ & $8(4-21,5)$ & $5(1,5-7,5)$ & $5(2-10)$ \\
\hline
\end{tabular}

(Me(RIC)**: Mediana y Rango intercuartílico 
Odds Ratio con un intervalo de confianza del 95\%, se presentan crudos y ajustados por variables de confusión, según criterios estadísticos. Se utilizó un valor $\mathrm{p}<0,05$, como criterio para considerar significación estadística.

Los datos fueron analizados utilizando el software estadístico Stata/S.E. Versión 11.1.

\section{Aspectos Éticos}

Ambos consentimientos informados - para el empleador y los trabajadores-, fueron evaluados y aceptados por el Comité de Ética en Investigación en Seres Humanos de la Facultad de Medicina de la Universidad de Chile.

Se explicitó que la participación en el estudio era de carácter voluntaria, que no revestía riesgos en ningún sentido, y que los cuestionarios buscan capturar la percepción de exposición a un riesgo específico laboral. Se aseguró un tratamiento seguro y confidencial de los datos allí vertidos, los que se presentan de forma agrupada y sólo serán utilizados con fines investigativos.

\section{RESULTADOS}

La muestra final estuvo conformada por cuatro empresas del rubro transporte. Tres de ellas pertenecientes a la Zona Metropolitana y una a la Región de Coquimbo. La empresa denominada “1”, tiene

\section{Tabla 2.}

Promedio y desviación estándar para los puntajes de las dimensiones de Factores de Riesgo Psicosociales presentes en el lugar de trabajo.

\begin{tabular}{|c|c|c|c|c|c|c|}
\hline $\begin{array}{l}\text { Dimensiones y } \\
\text { Sub-dimensiones }\end{array}$ & Empresa 1 & Empresa 2 & Empresa 3 & Empresa 4 & Total & $\begin{array}{c}\text { Referencia } \\
\text { Nacional }\end{array}$ \\
\hline EXIGENCIAS PSICOLÓGICAS & $62,2 \pm 13,9$ & $66,6 \pm 13,2$ & $57,7 \pm 11,9^{*}$ & $52,0 \pm 12,5^{*}$ & $56,7 \pm 13,6^{*}$ & $64,3 \pm 14,8$ \\
\hline Exigencias cuantitativas & $42,9 \pm 18,8$ & $48,8 \pm 16,8$ & $32,1 \pm 15,0$ & $15,0 \pm 16,3$ & $27,6 \pm 20,9$ & \\
\hline Exigencias cognitivas & $64,7 \pm 15,6$ & $68,5 \pm 12,2$ & $61,8 \pm 13,2$ & $61,7 \pm 17,5$ & $62,9 \pm 15,9$ & \\
\hline Exigencias emocionales & $60,8 \pm 22,4$ & $72,8 \pm 24,7$ & $55,8 \pm 20,2$ & $45,6 \pm 24,0$ & $53,3 \pm 24,2$ & \\
\hline \multicolumn{7}{|l|}{ Exigencias de } \\
\hline esconder emociones & $46,7 \pm 31,1$ & $52,9 \pm 26,8$ & $43,8 \pm 30,3$ & $41,9 \pm 32,6$ & $44,2 \pm 31,4$ & \\
\hline Exigencias sensoriales & $93,5 \pm 9,8$ & $89,9 \pm 12,8$ & $94,6 \pm 9,7$ & $97,0 \pm 6,5$ & $95,2 \pm 8,9$ & \\
\hline $\begin{array}{l}\text { TRABAJO ACTIVO Y } \\
\text { DESARROLLO DE HABILIDADES }\end{array}$ & $36,6 \pm 15,7$ & $44,6 \pm 14,2^{*}$ & $30,9 \pm 9,6^{*}$ & $33,4 \pm 13$ & $34,2 \pm 13,4$ & $33,9 \pm 13,1$ \\
\hline Influencia en el trabajo & $47,8 \pm 24,4$ & $53,1 \pm 21,6$ & $47,3 \pm 19,6$ & $49,4 \pm 27,2$ & $48,8 \pm 24,5$ & \\
\hline Posibilidades de desarrollo & $29,8 \pm 23,9$ & $30,8 \pm 21,9$ & $17,1 \pm 17,2$ & $26,9 \pm 18,5$ & $25,4 \pm 20,2$ & \\
\hline \multicolumn{7}{|l|}{ Control sobre el tiempo } \\
\hline de trabajo & $58,0 \pm 22,9$ & $69,5 \pm 23,8$ & $56,2 \pm 17,0$ & $58,4 \pm 23,7$ & $58,6 \pm 22,2$ & \\
\hline Sentido del trabajo & $9,2 \pm 16,2$ & $18,7 \pm 21,3$ & $4,4 \pm 9,5$ & $3,6 \pm 9,4$ & $6,1 \pm 13,0$ & \\
\hline Integración con la empresa & $36,9 \pm 28,2$ & $45,2 \pm 24,5$ & $28,5 \pm 20,3$ & $28,9 \pm 22,7$ & $31,7 \pm 24,0$ & \\
\hline $\begin{array}{l}\text { APOYO SOCIAL Y } \\
\text { CALIDAD DEL LIDERAZGO }\end{array}$ & $33,7 \pm 14,4$ & $42,3 \pm 19,3^{*}$ & $28,8 \pm 14,1^{*}$ & $28,1 \pm 14,2^{*}$ & $30,6 \pm 15,2^{*}$ & $32,6 \pm 15,1$ \\
\hline Previsibilidad & $40,9 \pm 25,5$ & $50,5 \pm 30,3$ & $39,1 \pm 26,1$ & $34,6 \pm 26,6$ & $38,2 \pm 26,8$ & \\
\hline Claridad de rol & $17,6 \pm 15,9$ & $18,5 \pm 20,4$ & $15,4 \pm 14,0$ & $13,4 \pm 15,3$ & $15,2 \pm 15,6$ & \\
\hline Conflicto de rol & $41,6 \pm 20,9$ & $53,6 \pm 26,8$ & $40,0 \pm 20,9$ & $32,6 \pm 21,1$ & $37,9 \pm 22,2$ & \\
\hline Calidad de liderazgo & $35,0 \pm 25,3$ & $57,2 \pm 30,0$ & $37,9 \pm 25,6$ & $30,4 \pm 24,7$ & $35,2 \pm 26,3$ & \\
\hline Apoyo social de compañeros & $31,0 \pm 26,0$ & $33,3 \pm 28,2$ & $25,9 \pm 19,4$ & $22,6 \pm 22,1$ & $26,0 \pm 23,1$ & \\
\hline Apoyo social de superiores & $31,3 \pm 28,3$ & $56,4 \pm 27,4$ & $33,3 \pm 28,5$ & $30,9 \pm 28,0$ & $33,5 \pm 28,8$ & \\
\hline Posibilidades de relación social & $49,2 \pm 27,0$ & $37,5 \pm 26,5$ & $23,4 \pm 22,7$ & $42,4 \pm 29,6$ & $38,9 \pm 28,9$ & \\
\hline Sentimiento de grupo & $21,6 \pm 23,1$ & $31,4 \pm 27,4$ & $15,5 \pm 15,6$ & $15,6 \pm 18,9$ & $18,0 \pm 20,3$ & \\
\hline $\begin{array}{l}\text { COMPENSACIONES EN } \\
\text { EL TRABAJO }\end{array}$ & $45,8 \pm 20,3$ & $48,3 \pm 20,7$ & $36,4 \pm 19,1^{*}$ & $36,8 \pm 20.0^{*}$ & $39,5 \pm 20,3^{*}$ & $42,1 \pm 20,8$ \\
\hline Inseguridad & $51,8 \pm 27,6$ & $47,8 \pm 30,8$ & $40,3 \pm 25,6$ & $42,8 \pm 28,3$ & $44,6 \pm 27,9$ & \\
\hline Estima & $39,2 \pm 21,5$ & $48,8 \pm 18,9$ & $32,4 \pm 21,5$ & $31,3 \pm 21,4$ & $34,5 \pm 21,8$ & \\
\hline DOBLE PRESENCIA & $27,1 \pm 17,1^{*}$ & $30,7 \pm 17,0^{*}$ & $31,1 \pm 16,8^{*}$ & $28,8 \pm 13,7^{*}$ & $29,2 \pm 15,5^{*}$ & $43,7 \pm 21,6$ \\
\hline
\end{tabular}

Fuente: Alvarado et al., 2009.

*: $p<0,05$, implica significación estadística respecto de la referencia nacional aportada por la fuente citada. su centro de operaciones en Santiago, y cuenta con tres faenas, todas ellas dedicadas al transporte de carga. La empresa denominada "2", localizada en la Zona Metropolitana, tiene sólo una faena destinada al transporte de carga. La empresa denominada “ 3 " es de la $4^{\circ}$ región, cuenta con 15 faenas destinadas en su totalidad al transporte de pasajeros. La empresa denominada "4", ubicada en Santiago, cuenta con 15 faenas de las cuales 9 fueron incluidas por criterio económico y de factibilidad de acceso; todas ellas dedicadas al transporte de pasajeros.

Se reclutaron 356 sujetos, en que la totalidad fue hombre (no existian mujeres contratadas en las empresas que decidieron participar). Destaca el promedio de horas trabajadas al día en la muestra general que fue de 11,4 \pm 3,8 horas, y a la semana de 51,9 $\pm 24,8$ horas, siendo superior a lo establecido legalmente (45 horas) (Ver Tabla 1). Respecto de las dimensiones y sub-dimensiones de los Factores de Riesgo Psicosociales presentes en el lugar de trabajo, la muestra de conductores analizada presenta en general promedios de exposición más bajos que los de la referencia nacional, salvo "trabajo activo y desarrollo de habilidades" que es 0,3 puntos superior, tal como se muestra en la Tabla 2 en donde se detallan los promedios y desviaciones estándar de cada una de las empresas participantes, y su comparación con la referencia.

Destaca "exigencias psicológicas" como la dimensión con promedio más alto, le siguen "compensaciones" y "trabajo activo y desarrollo de habilidades". Todas, salvo "trabajo activo y desarrollo de habilidades", presentan diferencias estadísticamente significativas respecto de la referencia nacional, siendo más altas para esta última en "doble presencia” (14,5 puntos) y “exigencias psicológicas" (7,6 puntos).

A continuación, en el Gráfico 1 se muestran los niveles de riesgo de exposición según terciles, evidenciando que en el nivel de alto riesgo (rojo) las dimensiones de "compensaciones en el trabajo" y de "apoyo social y calidad de liderazgo" son las que concentran más población sobre el percentil 66.

Los conductores de las empresas de transporte participantes autorreportan en un 93,8\% encontrarse "sin sospecha de alteraciones de salud mental (ausencia de psicopatología)"; mientras que un 3,1\% presenta resultados compatibles con "sospecha de psicopatología sub-umbral", e igual cifra muestra "hallazgos indicativos de alteraciones psicopatológicas". En el Gráfico 2 se desprende la distribución porcentual de estas categorias desagregadas por empresa, en donde destaca la número 2 por la suma de las categorías de "sospecha" y "hallazgos de psicopatología", resultando un 26,9\% de sus conductores afectados.

Respecto de la siniestralidad laboral de tránsito con participación directa e indirecta de los conductores pertenecientes a las empresas incluidas en el estudio, la empresa 2 es la que presenta la mayor tasa de accidentes de tránsito (Tabla 3).

Para evaluar la correlación existente entre las dimensiones y sub-dimensiones del ISTAS 21 y el puntaje GHQ-12 se utilizó la correlación de 


\section{Gráfico 1.}

Prevalencia de exposición a Factores de Riesgo Psicosociales presentes en el lugar de trabajo, según nivel de riesgo. Conductores de empresas de transporte de carga y pasajeros, urbanos e interurbanos, 2013.

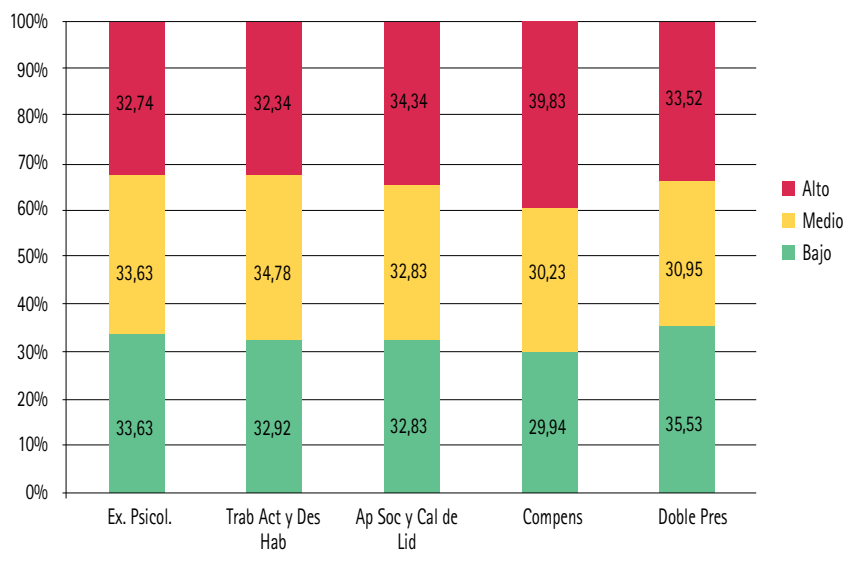

Gráfico 2.

Interpretación del Cuestionario de Salud de Goldberg GHQ-12. Conductores de empresas de transporte de carga y pasajeros, urbanos e interurbanos, 2013.

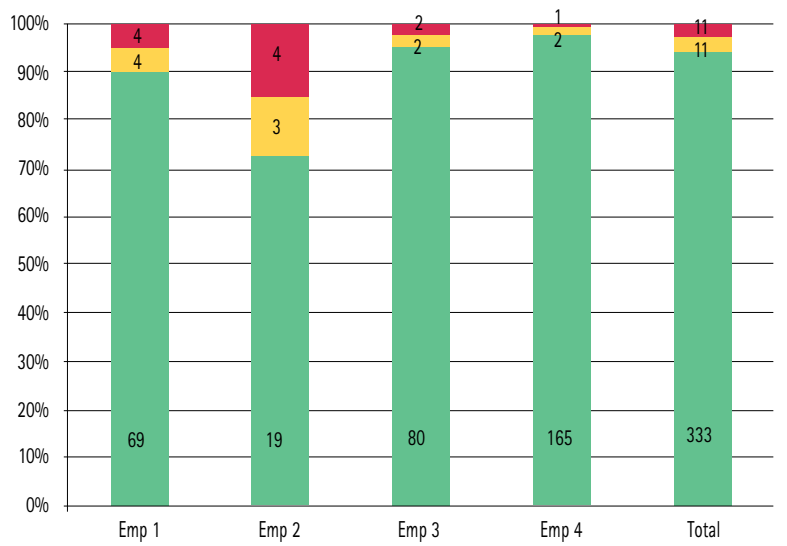

- Indicativos de presencia de psicopatología Sospecha de psicopatología subumbral \# Ausencia de psicopatología

Tabla 3.

Siniestralidad laboral (accidentes de tránsito).

\begin{tabular}{|c|c|c|c|c|c|}
\hline & Empresa & Empresa & Empresa & Empresa & 4 Total \\
\hline Vúmero de accidentes de tránsito & 74 & 48 & 43 & 47 & 230 \\
\hline Sumatoria de años de exposición & 404 & 155 & 1057 & 925 & 2541 \\
\hline $\begin{array}{l}\text { Tasa de Incidencia de accidentes } \\
\text { de tránsito }\end{array}$ & 0,18 & 0,31 & 0,04 & 0,05 & 0,09 \\
\hline $\begin{array}{l}\text { Conductores que han protagonizado } \\
\text { accidentes con lesionado grave (\%) }\end{array}$ & 14,3 & 26,9 & 15,7 & 9,0 & 13,0 \\
\hline $\begin{array}{l}\text { Conductores que han protagonizado } \\
\text { accidentes fatales }(\%)\end{array}$ & 3,9 & 0 & 4,8 & 1,8 & 2,8 \\
\hline
\end{tabular}

Spearman (ver Tabla 4), dado que ambas variables distribuyen de forma no normal.

Las 5 dimensiones presentan correlaciones intermedias estadísticamente significativas, siendo "exigencias psicológicas" la del valor más elevado $(\mathrm{r}=0,438, \mathrm{p}<0,001)$ y la más baja para "trabajo activo y desarrollo de habilidades" $(r=0,238, \mathrm{p}<0,001)$.

Respecto de las sub-dimensiones, se tiene que sólo dos resultaron no tener correlación estadísticamente significativa: "influencia en el trabajo" $(\mathrm{r}=0,052, \mathrm{p}=0,329)$ y "control sobre los tiempos de trabajo" $(\mathrm{r}=0,089, \mathrm{p}=0,099)$.
Tabla 4.

Correlación entre las dimensiones y sub-dimensiones del ISTAS-21 y el puntaje GHQ-12.

\begin{tabular}{lcc} 
Dimensión / Sub-dimensión & $r$ Spearman & valor p \\
EXIGENCIAS PSICOLÓGICAS & 0,438 & $<0,001^{*}$ \\
Exigencias cuantitativas & 0,402 & $<0,001^{*}$ \\
Exigencias cognitivas & 0,209 & $<0,001^{*}$ \\
Exigencias emocionales & 0,433 & $<0,001^{*}$ \\
Exigencias de esconder emociones & 0,279 & $<0,001^{*}$ \\
Exigencias sensoriales & $-0,152$ & $0,004^{*}$ \\
TRABAJO ACTIVO Y DESARROLLO DE HABILIDADES & 0,238 & $<0,001^{*}$ \\
Influencia en el trabajo & 0,052 & 0,329 \\
Posibilidades de desarrollo & 0,146 & $0,006^{*}$ \\
Control sobre el tiempo de trabajo & 0,089 & 0,099 \\
Sentido del trabajo & 0,258 & $<0,001^{*}$ \\
Integración con la empresa & 0,260 & $<0,001^{*}$ \\
APOYO SOCIALY CALIDAD DEL LIDERAZGO & 0,399 & $<0,001^{*}$ \\
Previsibilidad & 0,224 & $<0,001^{*}$ \\
Claridad de rol & 0,208 & $<0,001^{*}$ \\
Conflicto de rol & 0,381 & $<0,001^{*}$ \\
Calidad de liderazgo & 0,303 & $<0,001^{*}$ \\
Apoyo social de compañeros & 0,228 & $<0,001^{*}$ \\
Apoyo social de superiores & 0,249 & $<0,001^{*}$ \\
Posibilidades de relación social & 0,157 & $0,003^{*}$ \\
Sentimiento de grupo & 0,252 & $<0,001^{*}$ \\
COMPENSACIONES EN EL TRABAJO & 0,424 & $<0,001^{*}$ \\
Inseguridad & 0,338 & $<0,001^{*}$ \\
Estima & 0,387 & $<0,001^{*}$ \\
DOBLE PRESENCIA & 0,251 & $<0,001^{*}$ \\
* valor p < 0,05 & & \\
\hline
\end{tabular}

Tabla 5.

Correlación entre las dimensiones y sub-dimensiones del ISTAS-21 y accidentes de tránsito.

$\begin{array}{lcc}\text { Dimensión / Sub-dimensión } & r \text { Spearman } & \text { valor p } \\ \text { EXIGENCIAS PSICOLÓGICAS } & 0,147 & 0,007^{*} \\ \text { Exigencias cuantitativas } & 0,200 & 0,001^{*} \\ \text { Exigencias cognitivas } & 0,005 & 0,920 \\ \text { Exigencias emocionales } & 0,131 & 0,014^{*} \\ \text { Exigencias de esconder emociones } & 0,077 & 0,153 \\ \text { Exigencias sensoriales } & -0,108 & 0,044^{*} \\ \text { TRABAIO ACTIVO Y DESARROLLO DE HABILIDADES } & 0,042 & 0,451 \\ \text { Influencia en el trabajo } & -0,054 & 0,317 \\ \text { Posibilidades de desarrollo } & 0,001 & 0,986 \\ \text { Control sobre el tiempo de trabajo } & 0,028 & 0,606 \\ \text { Sentido del trabajo } & 0,175 & 0,001^{*} \\ \text { Integración con la empresa } & 0,081 & 0,134 \\ \text { APOYO SOCIAL Y CALIDAD DEL LIDERAZGO } & 0,149 & 0,007^{*} \\ \text { Previsibilidad } & 0,098 & 0,068 \\ \text { Claridad de rol } & 0,014 & 0,794 \\ \text { Conflicto de rol } & 0,189 & 0,001^{*} \\ \text { Calidad de liderazgo } & 0,142 & 0,007^{*} \\ \text { Apoyo social de compañeros } & 0,078 & 0,146 \\ \text { Apoyo social de superiores } & 0,108 & 0,043^{*} \\ \text { Posibilidades de relación social } & 0,030 & 0,575 \\ \text { Sentimiento de grupo } & 0,126 & 0,019^{*} \\ \text { COMPENSACIONES EN EL TRABAJO } & 0,248 & <0,001^{*} \\ \text { Inseguridad } & 0,193 & 0,001^{*} \\ \text { Estima } & 0,211 & 0,001^{*} \\ \text { DOBLE PRESENCIA } & 0,088 & 0,101\end{array}$

* valor $p<0,05$

Para evaluar la correlación existente entre las dimensiones y subdimensiones del ISTAS 21 y el número de accidentes de tránsito se utilizó también correlación de Spearman (ver Tabla 5).

Tres dimensiones presentan correlaciones bajas pero estadísticamente significativas, siendo "compensaciones" la del valor más elevado $(\mathrm{r}=$ $0,248, \mathrm{p}<0,001)$, le siguen "apoyo social y calidad del liderazgo" $(\mathrm{r}=$ $0,149, \mathrm{p}=0,007) \mathrm{y}$ “exigencias psicológicas” ( $\mathrm{r}=0,147, \mathrm{p}=0,007)$. 
Tabla 6.

Asociación cruda y ajustada según variables de confusión entre Factores de Riesgo Psicosociales presentes en el lugar de trabajo y GHQ-12.

\begin{tabular}{|c|c|c|c|c|c|c|}
\hline Dimensión & \multicolumn{3}{|c|}{ Modelo Crudo } & \multicolumn{3}{|c|}{ Modelo Ajustado } \\
\hline Trabajo Act y Des de Habilidades & $0,04 \pm 0,01$ & 0,001 & $(0,03-0,06)$ & $0,04 \pm 0,01$ & 0,001 & $(0,02-0,05)$ \\
\hline Compensaciones & $0,04 \pm 0,01$ & 0,001 & $(0,03-0,05)$ & $0,04 \pm 0,01$ & 0,001 & $(0,03-0,04)$ \\
\hline Doble Presencia & $0,03 \pm 0,01$ & 0,001 & $(0,02-0,04)$ & $0,03 \pm 0,01$ & 0,001 & $(0,02-0,04)$ \\
\hline
\end{tabular}

En la Tabla 6 se muestra el análisis de asociación bivariado entre las dimensiones de los factores de riesgo psicosociales y percepción de salud (GHQ-12) y, multivariado ajustado por las variables de confusión: nivel educacional, situación de pareja, zona de desempeño y tipo de transporte (si es de carga o pasajeros), las que se seleccionaron considerando criterios estadísticos, de modo de lograr un modelo parsimonioso y con el mejor coeficiente de correlación $\left(\mathrm{r}^{2}\right)$.

Como se aprecia en la tabla, luego de ajustar por las variables de confusión definidas, las asociaciones se mantuvieron significativas con un valor $\mathrm{p}<0,001$ y sólo redujeron el aumento de la puntuación de GHQ en un punto, tanto para la asociación con "exigencias psicológicas" como para "apoyo social y calidad del liderazgo".

La participación en accidentes de tránsito por parte del conductor de la empresa participante, ya sea directa o indirectamente, fue considerada en el cuestionario como variable proxy de la siniestralidad laboral -de tránsito-, y para efecto de este análisis en particular, fue dicotomizada (no participa/participa en accidentes de tránsito como conductor de la empresa en cuestión).

En la Tabla 7 se muestran los análisis de asociación bivariado entre las dimensiones de los factores de riesgo psicosociales y los accidentes de tránsito, y el multivariado, en que las variables de confusión incluidas fueron: nivel educacional, situación de pareja, zona de desempeño, tipo de transporte (si es de carga o pasajeros), horario de trabajo y años trabajando como conductor en la empresa.

\section{Tabla 7.}

Asociación cruda y ajustada según variables de confusión entre Factores de Riesgo Psicosociales presentes en el lugar de trabajo y Siniestralidad de tránsito laboral.

$\begin{array}{lcc}\text { Dimensión } & \text { OR Crudo (IC 95\%) } & \text { OR Ajustado (IC 95\%) } \\ \text { Exigencias psicológicas } & 1,02(1,00-1,03)^{*} & 0,99(0,97-1,01) \\ \text { Trabajo Activo y Desarrollo de Habilidades } & 1,00(0,99-1,02) & 1,00(0,98-1,02) \\ \text { Apoyo Social y Calidad del Liderazgo } & 1,02(1,00-1,04)^{*} & 1,01(0,99-1,03) \\ \text { Compensaciones } & 1,03(1,01-1,04)^{*} & 1,02(1,01-1,04)^{*} \\ \text { Doble Presencia } & 1,01(0,99-1,03) & 1,01(0,99-1,03)\end{array}$

Ajustado por: educación, situación de pareja, zona de desempeño, tipo de transporte, horarios de trabajo y años trabajando en la empresa como conductor. * valor $p<0,05$.

Como se aprecia en la tabla anterior, luego de ajustar por variables de confusión, la asociación se mantuvo significativa sólo para la dimensión "compensaciones" (con valores $\mathrm{p}<0,05$ ).

Ahora bien, respecto de las intervenciones propuestas se releva la importancia de proponer que éstas sean organizacionales, preventivas y específicas. Éstas fueron consensuadas con las empresas participantes, de modo de que sean factibles de realizar. Entre ellas, destacan:

- exigencias de trabajo razonables en cuanto a la carga;

- enriquecer el contenido del trabajo para disminuir el bajo sentido del trabajo, la baja influencia sobre éste y el conflicto de rol;
- que el trabajo conlleve autonomía;

- rediseño de procesos, tareas y perfiles de cargo, que permitan aplicar y desarrollar las habilidades y conocimientos de los conductores;

- desarrollo de habilidades de comunicación y coordinación efectiva en los supervisores para minimizar malentendidos y conflictos;

- desarrollo de un estilo de liderazgo que privilegie la confianza y autonomía responsable en los conductores;

- que faciliten el trabajo cooperativo mediante la ayuda de superiores y compañeros en la realización de las tareas, lo que reduce la baja calidad de liderazgo, baja claridad de rol, baja estima y altas exigencias de esconder emociones;

- con salarios justos;

- con ritmos y dotaciones razonables que disminuyan la doble presencia, falta de control sobre los tiempos e inseguridad sobre la jornada y horario;

- que genere estabilidad laboral;

- que "informe sobre lo cotidiano" disminuyendo inseguridad y potenciando la integración;

- que promueva una cultura de seguridad laboral;

- que cuente con compensaciones justas.

\section{DISCUSIÓN}

Los promedios de las dimensiones de factores de riesgo psicosociales presentes en el lugar de trabajo en los conductores resultaron ser estadísticamente significativos más bajos que los de la referencia nacional, salvo "trabajo activo y desarrollo de habilidades". Las dimensiones de mayor prevalencia de exposición resultaron ser "exigencias psicológicas", "compensaciones" y "trabajo activo y desarrollo de habilidades". Y las que concentraron más población en el nivel de riesgo alto, fueron "compensaciones" y "apoyo social y calidad de liderazgo". Por su parte, las sub-dimensiones más prevalentes fueron "exigencias sensoriales", "exigencias cognitivas" y "control sobre el tiempo de trabajo." El 93,8\% de la muestra resultó "sin sospecha de alteraciones de salud mental". La tasa de incidencia de accidentes de tránsito resultó variada entre las empresas.

Se determinó una correlación positiva intermedia, pero significativa entre cada una de las dimensiones del ISTAS 21 y el GHQ-12; por otro lado, se determinó una correlación positiva baja, pero significativa, para tres dimensiones del ISTAS 21 ("exigencias psicológicas", "apoyo social y calidad del liderazgo" y "compensaciones”) y los accidentes de tránsito.

Entre la exposición a factores de riesgo psicosociales presentes en el lugar de trabajo y la percepción de salud, se demostró una asociación significativa para todas las dimensiones, las cuales se mantuvieron aun después de ajustar por las variables confusoras. 
Entre la exposición a factores de riesgo psicosociales presentes en el lugar de trabajo y los accidentes de tránsito, se demostró una asociación significativa sólo para compensaciones, luego de ajustar por variables de confusión.

Tanto para esta muestra de conductores como para la referencia nacional, la dimensión "exigencias psicológicas” resultó ser la más prevalente, pero en el segundo y tercer lugar hay diferencias, ya que en este estudio "compensaciones" y "trabajo activo y desarrollo de habilidades" ocuparon el $2^{\circ}$ y $3^{\circ}$ lugar respectivamente, a diferencia de la referencia en que el segundo lugar lo ocupó "doble presencia" y el $3^{\circ}$ "compensaciones", en donde el 71,3\% de la muestra era mujer.

Entre las fortalezas de este estudio se cuentan que se realizó sobre conductores de transporte de carga y de pasajeros, urbanos e interurbanos, aportando con información relevante, en términos exposición a factores de riesgo psicosociales presentes en el lugar de trabajo para este rubro por primera vez en nuestro país, incluso antes de la entrada en vigencia del Protocolo de Vigilancia ministerial; no hubo rechazo a participar una vez realizada la charla de sensibilización; se utilizaron cuestionarios validados en Chile y en el resto del mundo (GHQ-12), lo que nos permite la comparación de los resultados; y que las intervenciones propuestas a cada empresa, en virtud de sus resultados, son frutos del trabajo consensuado con cada una de ellas, lo que genera participación directa, compromiso y movilización hacia la acción.

Entre las limitaciones destaca la acotada validez interna del estudio, dadas las dificultades en conseguir la aceptación a participar por parte de las empresas contactadas desde la mutualidad, lo que determinó que sólo se incluyeran cuatro, introduciendo así un sesgo de selección.

Dado que se utilizó la versión media del ISTAS 21 y no la larga, no se pudieron comparar los valores de las sub-dimensiones de la muestra global y por empresa con los de la referencia nacional de modo de precisar mejor los focos a intervenir. Cabe destacar, además, que existen diferencias entre los promedios y desviaciones estándar de las versiones media y larga, teniendo esta última mayor representatividad nacional (ya que incluye varias actividades económicas y no sólo servicios). No se usó la versión completa dado que ésta obliga informar los resultados a la Superintendencia de Seguridad Social, reduciendo así las posibilidades de lograr participación por parte de los representantes legales de las empresas.

Esta investigación no hace más que confirmar la abundante evidencia que existe referente a este tema, y que da cuenta, de modo transversal, de las elevadas prevalencias de exposición a estos factores de riesgo laborales específicos, las que potencialmente no sólo afectan la productividad, sino que también generan daño o exacerban alteraciones fisiológicas preexistentes en los trabajadores expuestos, particularmente trastornos músculoesqueléticos y alteraciones de salud mental; así como también aumentan el ausentismo, presentismo, enfermedades profesionales $\mathrm{y}$ accidentes del trabajo.

Un grupo especial son los conductores de vehículos de transporte, quienes, además, por el trabajo que desempeñan pueden causar daños a terceros. Es por ello que, ya identificados los riesgos psicosociales en ellos, cuantificados éstos y estimadas las asociaciones con la percepción de salud y siniestralidad (de tránsito) laboral, procede intervenir sobre aquellos agentes partiendo por los más prioritarios, con intervenciones de enfoque organizacional, integral y participativo, cuyos resultados sean evaluados y contrastados con la situación previa, lo que constituye una línea investigativa a desarrollar en el futuro. 
1. Moreno B, Báez C. Factores y riesgos psicosociales, formas, consecuencias, medidas y buenas prácticas [en línea]. Madrid: INSHT; 2010 [consultado 23 sept 2013]. Disponible en: http://www.insht.es/InshtWeb/Contenidos/ Documentacion/PUBLICACIONES\%20PROFESIONALES/factores $\% 20$ riesgos\%20psico.pdf

2. Benavides F, Gimeno D, Benach J, Martínez J, Jarque S, Berra A. Descripción de los factores de riesgo psicosocial en cuatro empresas. Gaceta Sanit. 2002;16(3):222-29.

3. Peiró J. Desencadenantes del estrés laboral. Madrid: Eudema; 1993.

4. Agencia Europea para la Seguridad y Salud en el Trabajo. Trabajemos contra el estrés [en línea]. Luxemburgo: OSHA; 2002. (Magazine, 5). [consultado 22 dic 2013].Disponible en: https://osha.europa.eu/es/publications/magazine/5 [

5. Karasek R. The impact of the work environment on life outside the job. [Tesis doctoral]. Cambridge: MIT; 1976.

6. Karasek R, Theorell T. Healthy Work, Stress, Productivity and the Reconstruction of Working Life. New York: Basic Book; 1990.

7. Schnall $P$, Landsbergis $P$, Baker D. Job strainand cardiovascular disease. Ann Review Public Health. 1994;15:381-411.

8. Bongers $\mathrm{P}, \mathrm{De}$ Winter $\mathrm{C}$, Kompier M, Hildebrandt V. Psychological factors at work and musculoskeletal disease. Scand J Work Environ Health. 1993;19:297-312.

9. Leka S, Griffiths A, Cox T. Work Organization and Stress. Geneva: WHO; 2003. (Protecting Workers Health Series; 3 )

10. Cox T, Rial-Gonzalez E. Risk management, psychosocial hazards and work stress. Psychological Stress at Work. Helsinki: Finnish Institute of Occupational Health; 2000.

11. Siegrist J. Adverse health effects of high effort-low reward conditions. J Occup Health Psychol. 1996;1:27-41.

12. European Commission. Health and Safety at Work in Europe (1999-2007). A statistical portrait. Luxemburg: Publications Office of the European Union; 2010.

13. European Agency for Safety and Health at Work. Occupational safety and health in figures: Occupational safety and health in the transport sector. An overview. Luxemburg: Publications Office of the European Union; 2011.

14. Kompier M, Di Martino V. Review of bus driver's occupational stress and stress prevention. Stress Medicine. 1995; 11(4):253-262.

15. Tse J, Flin R, Mearns K. Bus driver well-being review: 50 years of research. In: transportation Research. Part F9. Edinburg: Elsevier; 2006. p.89-114.

16. Aronsson $G$, Rissler A. Psycho-physiological stress reactions in female and male urban bus drivers. J Occup Health Psychol. 1998;3:122-129.

17. Mira M, Espinos M, Morales P, Segarra I, Sisa M, López L, et al. Vigilancia de la salud en trabajadores que refieren sintomas de estrés percibidos, cambios en biomarcadores de activación del eje hipofisosuprarrenal. Barcelona: Servei de Prevención de TMB; 2007.

18. Salas $C$, Meliá J. Evaluación de riesgos psicosociales y factores psicosociales que afectan a la probabilidad de accidente en una empresa de transporte urbano de economía social[en línea]. Valencia: Universidad de Valencia; 2004 [consultado18 nov 2013].Disponible en: http://www.uv.es/meliaj/Papers/ 2004SalasMelia.pdf
19. Couto M, Lawoko S. Burnout, workplace and social support among drivers and conductors in the road passenger transport sector in Maputo City, Mozambique. J Occup Health. 2011;53:214-221.

20. Ragland D, Greiner B, Yen I, Fisher J. Occupational stress factors and alcoholrelated behavior in urban transit operators. Alcohol Clin Exp Res. 2000; 24:1011-19.

21. Gardell B, Aaronson G, Barkloff K. The Working Environment for Local Public Transport Personnel. Stockholm: The Swedish Work Environmental Fund; 1982.

22. Cunradi C, Greiner B, Ragland D, Fisher J. Alcohol, Stress-Related Factors, and Short-Term Absenteeism Among Urban Transit Operators. Journal of Urban Health: Bulletin of the New York Academy of Medicine [on line]. 2005;82(1):43-57 [consultado 23 nov 2013].Disponible en: http://cmbi.bjmu. edu.cn/news/report/2004/Urban/view/4.pdf

23. Issever $H$, Onen L, Sabuncu $H$, Altunkaynak 0 . Personality characteristics, phychological symptoms and anxiety levels of drivers in charge of urban transportation is Istanbul. Occup Med. 2002;52(6):297-303.

24. Budd T. Violance of work: New findinggs from the 2000 British crime survey. London: Health \& Safety Executive; 2001.

25. Bongers $P$, de Winter $C$, Kompier $M$, Hildebrandt H. Psychosocial factors at work and musculoskeletal disease. Scandinavian Journal of Work, Environment and Health 1993;19:297-312.

26. Chile Ministerio de Salud, Dirección del Trabajo e Instituto de Seguridad Laboral. Primera Encuesta Nacional de Empleo, Trabajo, Salud y Calidad de Vida de los Trabajadores y Trabajadoras en Chile (ENETS 2009-2010) [en línea]. Santiago: MINSAL; 2011 [consultado 21 ago 2013].Disponible en: http://www. google.cl/url?sa $=$ t\&rct=j\&q=\&esrc=s\&source $=$ web $\& c d=1 \&$ ved $=0 C C k Q F j A A$ \&url $=$ http $\% 3 \mathrm{~A} \% 2 \mathrm{~F} \% 2 \mathrm{Fwww.eligevivirsano.cl} \% 2 \mathrm{Fwp}$-content $\% 2$ Fuploads $\% 2$ F2012\%2F01\%2FInforme-Encuesta-ENETS-2009-2010.pdf\&ei=lyCBU_ SzHvi-sQSmloCoBg\&usg=AFOjCNE9imNVOPnA5Bd7gDFR_pMORDWq WA\&bvm =bv.67720277,d.cGU

27. Pérez-Franco J Distribución del riesgo psicosocial laboral el Chile. Rev Chil Salud Pública [en línea]. 2014;18(1):52-60. Disponible en: http://www.revistasaludpublica.uchile.cl/index.php/RCSP/article/viewFile/30755/32501 [consultado 02 mar 2014].

28. Olivares V, Jélvez $C$, Mena L, Lavarello J. Estudios sobre Burnout y Carga Mental en Conductores del Transporte Público de Chile (Transantiago). Cienc Trab [en línea]. 2013;15(48):173-78 [consultado14 mar 2014]. Disponible en: http://dx.doi.org/10.4067/S0718-24492013000300011 [Consultado el 14 de marzo de 2014].

29. Neisa C, Rojas Y. Fatiga laboral, accidentes e incidentes laborales en los conductores de carga pesada de una empresa Transportista de la Ciudad de Yopal. Cuadernos Hispanoamericanos de Psicologia [en línea]. 2009;10(1):7-21 [consultado 14 mar 2014].Disponible en: http://www.uelbosque. edu.co/sites/default/ files/publicaciones/revistas/cuadernos_hispanoamericanos_psicologia/ volumen10_numero1/articulo_1.pdf

30. Kaur S. Detección de la fatiga en la conducción [en línea]. Querétaro: Instituto Mexicano del Transporte; 2014. [consultado 03 ene 2014]. Disponible en: http://www.imt.mx/Espanol/Publicaciones/pubtec/ 